\title{
MEMORIA DE ACCIÓN EDUCATIVA
}

\section{EDUCATIONAL ACTION REPORT}

Referencia de reseña

Reynoso Tavico, T. V. (2021). Memoria Acción Educativa. Revista Científica del Sistema de Estudios de Postgrado de la Universidad de San Carlos de Guatemala, 4(1), 125-133. DOI: https://doi.org/10.36958/sep.v4i1.80

\author{
Mtra. Viviana Reynoso Tavico \\ licda.vivianareytavi@gmail.com \\ 0000-0002-0284-4827 \\ Maestría en Docencia Universitaria \\ Universidad de San Carlos de Guatemala
}

Fecha de recepción: 01/07/2021

\section{RESUMEN}

La presente reseña detalla acciones fundamentales dentro del proceso y hecho educativo que coadyuvaran la administración de cualquier centro educativo del sector oficial, privado o municipal del sistema educativo nacional; sin descartar el nivel superior, principalmente cuando los retos y desafíos por llevar y generar cambios significativos en los contextos más vulnerables de la nación guatemalteca. Especialmente con las diferentes problemáticas que a diario aquejan a una población mayoritaria al vivir en resiliencia desde diversas exceptivas de la vida. De igual forma el Maestro Afre comparte diversas experiencias de liderazgo al encauzar al personal a cargo, a fin de dirigir a un elemento importante del proceso de formación "los docentes", como entes indispensables que facilitan el aprendizaje de los estudiantes a cargo.

Por lo cual es de suma importancia tomar como referencia de las memorias de acción educativa, al proporcionar una serie reflexiones y acciones congruentes y pertinentes a la realidad nacional, especialmente con las secuelas que provocó la pandemia COVID-19 ante los desafíos actuales del proceso educativo de la nación; por lo que es indispensable orientar al administrador educativo y docentes de cualquier nivel de educación a tener conciencia, creatividad y humanidad para fomentar el aprendizaje; sino también el desaprender a fin de crear un verdadero ideal de formación, acorde a las necesidades, demandas y realidades del entorno social actual y propiciar mejoras a beneficio del bien común de la sociedad guatemalteca.

\section{PALABRAS CLAVE}

Reseña, cambios, aprendizaje, docente
Fecha de aceptación: 17/09/2021

\section{ABSTRACT}

This review details fundamental actions within the educational process and fact that will contribute to the administration of any educational center in the official, private or municipal sector of the national educational system; without ruling out the higher level, mainly when the challenges to carry and generate significant changes in the most vulnerable contexts of the Guatemalan nation. Especially with the different problems that affect a majority population on a daily basis as they live in resilience from various exceptions of life. Similarly, Master Afre shares various leadership experiences by channeling the personnel in charge, in order to direct an important element of the training process "the teachers", as indispensable entities that facilitate the learning of the students in charge.

Therefore, it is of the utmost importance to take as a reference the memories of educational action, by providing a series of reflections and actions that are consistent and pertinent to the national reality, especially with the consequences caused by the COVID-19 pandemic in the face of the current challenges of the educational process. of the nation; Therefore, it is essential to guide the educational administrator and teachers of any level of education to have awareness, creativity and humanity to promote learning; but also unlearning in order to create a true ideal of training, according to the needs, demands and realities of the current social environment and to promote improvements for the benefit of the common good of Guatemalan society.

\section{KEYWORDS}

Review, changes, learning, teacher 
El presente libro narra de manera pertinente la situación real que viven varios centros educativos públicos de nivel primario en el área rural, desde el desinterés de las autoridades en proporcionar a la población un servicio educativo de calidad, tal como lo indica la constitución política de la república de Guatemala en su artículo 74; al propiciar al estado las condiciones básicas para mejorar su modo de vida como un derecho no como un privilegio. Especialmente de los años 90, en donde la mayoría de los docentes y administradores educativos, afrontan diversas retos y desafíos al establecer una comunidad educativa acorde a las necesidades y demanda del entorno inmediato del cantón Xolacul, de Santa María, Nebaj, Quiché.

Explica de manera contundente la situación real de un docente en tiempos del conflicto armado interno, la situación socioeconómica de la población en servicio educativo, en donde se debe establecer mecanismos para forjar el desarrollo comunitario integral en un contexto histórico con secuelas a causa de diferentes flagelos sociales vivenciales hasta hoy en día persisten y estancan la evolución de la comunidad en mención. Por lo que a continuación se explicara las cuatro partes de este escrito, que proporciona acciones concretas para realizar un servicio educativo efectivo a los benefactores de los trabajadores del estado.

La primera parte del libro indica cómo fue la constitución organizacional de la Escuela Oficial Rural Mixta Cantón Xolacul, en donde Afre (2016) indica" la escuela fue apertura da en enero de 1982, con la autorización del supervisor correspondiente del NEPADE No. 12; en donde el Profesor Adrián Franco fue el primer director por dos días y por situaciones laborales lo sustituye el Prof. Miguel Ángel García Taracena e inicia su funcionamiento en la localidad de la iglesia católica de la comunidad." Con el tiempo se gestiona plazas 011 para la estabilidad de servicios profesionales de docentes, la primera plaza que asigna el Ministerio de Educación para la escuela en mención, quien la obtiene es la Profesora Amanda Leticia Padilla de Argueta, que es de origen de orientes específicamente de Chiquimula en donde toma posesión el dos de enero de 1985.

Mediante el apoyo de la docente en mención y compañeros del claustro en calidad de reubicados; iniciaron gestiones para comparar un predio legítimo de la comunidad de Xolacul; en donde se organizó con los miembros de la comunidad al tener liderazgo de don Jacinto Santiago de León convoca a los pobladores en realizar colectas conjuntamente con los docentes, al realizar la compra del inmueble con la finalidad de tener una infraestructura física propia de la entidad educativa; el cual propicia nuevos senderos de desarrollo integral de los habitantes de dicha comunidad.

A través de gestiones ante entes no estatales y gubernamentales los miembros del centro educativo logran la construcción de la escuela, que se ubica en la calle principal comunitaria con tres aulas, con el apoyo de la docente Feliciana Cedillo Bernal y aportes de la comunidad concretizan el pago del primer predio de la institución educativa. También mediante más trabaja en equipo del centro escolar obtiene la adquisición de otro predio para la establecer más específicos físicos para los niños, es fue gracias al apoyo del señor alcalde municipal José Fidel Mérida Molina y el Consejo de Desarrollo Urbano y Rural permitió la instauración de la dirección del este mismo conjuntamente con tres aulas más, en donde la colaboración de los padres fue indispensable el cual fue inaugurado en el mes de octubre del año 1995.

La gestión y autogestión comunitaria gesta el logro de diferentes proyectos en pro de 
sus habitantes, por lo que antes de iniciar el siglo XXI la comunidad y las autoridades municipales logran la compra de una cuerda de terreno con el apoyo del señor alcalde municipal profesor Pedro Raymundo y la gestión ante el Fondo de Inversión Social se adquieren dos en abril del año 2000. Destacar que en el año 2012 se logra un laboratorio de computación en centro educativo en mención; ya que fue una escuela modelo del futuro de parte del Ministerio de Educación, la gestión de tan magnífico proyecto fue del Prof. Aroldo Joaquín Morales López.

La segunda parte de la obra describe el marco filosófico de la institución en donde enmarca horizontes específicos para conducir a los pobladores al desarrollo integral, como parte de los objetivos de desarrollo sostenible especialmente el número 4, que busca dar calidad educativa a los usuarios, mediante el mejor proceso de cambio de un entorno social "LA EDUCCIÓN"; por lo que la Escuela Oficial Rural Mixta del Cantón Xolacul, del municipio de Santa María Nebaj del departamento de Quiché, se enfoca con su objetivo principal que es impartir una docencia pertinente a la realidad del entorno social, de manera organizada y técnica, a fin de orientar a la comunidad a su mejora integral.

En sus objetivos específicos establece diseñar una planificación docente con las exigencias y estrategias del currículo nacional base; de igual forma propiciar la participación de los padres de familia como eje fundamental de la formación de los estudiantes, sin dejar de orientar a la comunidad para impulsar desarrollo de diversos beneficios sociales comunitarios (Afre, 2016). Tener una atención de 400 estudiantes, al permitir el involucramiento de los progenitores del estudiantado, dotara una mejor consolidación académica en los ciudadanos presente de la comunidad en mención.
El tener claro el enfoque pedagógico a desempeñar en la institución, determina según la declaración de derechos humanos (1948)" la educación parte como un derecho universal para el desarrollo integral del ser humano, como ente de cambio en pro de su entorno inmediato, a fin de realizar diversas actividades que beneficien de forma conjunta e individual del ser humano como ente de cambio". Tener en consideración la importancia de forjar un proceso de enseñanza aprendizaje acorde a principios y valores integrales, en base a políticas, estrategias que encaucen a la niñez a mejorar su conocimiento cultural, científico, tecnológico y cultural según lo que establece el currículo nacional base del sistema educativo de Guatemala, en función de orientar a los habitantes a construir un mejor modo de vida. Dentro de las políticas del establecimiento educativo está en que el diseño de educativo se coherente con la reforma educativa del país para responder las necesidades contextuales del municipio de Santa maría Nebaj. De igual forma implementar la educación bilingüe intercultural, mediante distintos programas del estado, organizaciones no gubernativas que sumen a los diferentes procesos desarrollados en el ente educativo del cantón Xolacul.

En la tercera parte de la obra describe anécdotas del Maestro Afre en donde narra situaciones reales dentro de distintos centros educativos desde diferentes aspectos sociales, económicos, psibiológicos que influyen en el desarrollo integral de la comunidad educativa en mención, por lo que a continuación de enunciaran algunas situaciones y acciones que permiten a todo trabajador del estado pueda poner en practicar en cualquier escuela pública de la nación guatemalteca. Por mencionar, la selección de personal para el reglón 011 en la década de los 80 era muy riguroso, especialmente al someterse a exámenes básicos de matemática e idioma español, en donde los mejores punteos se 
tomaban en cuenta para la asignación de un puesto docente en cualquier departamento o municipio del país.

Por lo que el Maestro C. Fernando Afre en 1986 recibe mediante un telegrama presentarse ante el Ministerio de Educación, para recibir su nombramiento e ir atender a la comunidad educativa, designada por el ente encargado de la cartera en mención. El día 21 de abril de 1986, el docente inicia su aventura y ejercicio profesional en el Cantón Xolacul en la supervisión, en donde fue asignado director del establecimiento educativo por lo que venían muchos retos y desafíos. Y definitivamente tendría que iniciar con planes, estrategias de trabajo con el personal, equipo, mobiliarios infraestructura, formación docente, acompañamiento de autoridades, trabajar como docentes multigrado, era tiempo de establecer líneas de acción para forjar un mejor presente para la población en servicio.

Dentro de la experiencia profesional del Maestro Carlos Fernando Afre Arévalo se describe las acciones con aportes concretos para lidiar centros escolares del área rural y urbana de cualquier nivel educativo, incluso a nivel de educación superior desde el rol de administrador educativo y docente, en el cual se describen las siguientes acciones educativas:

Tener vocación docente para ejercer la labor educativa con deseo, proactividad, productividad sin excusa alguna, para impartir el pan del saber como si fuera el último día de vida, al tener visión de emprender un futuro prometedor a los niños y niñas que asisten con esfuerzos a la escuela, mediante el liderazgo y pasión de trabajo, no solo como un deber y obligación del servidor público, sino que incursionar responsablemente en pro de mayorías.
Establecer medios sanos para dirigir a un equipo de trabajo con retos y desafíos latentes en un establecimiento con carencias; en función de establecer acciones pertinentes que favorezca un servicio adecuado a los usuarios del centro escolar donde se labora. Especialmente al atender multigrados y consolidar acuerdos con docentes reubicados, en dar una docencia favorable a un contexto bilingüe. A esto se le llama en situación administrativa integración del personal, el cual promueve una cultura organizacional funcional para el logro de objetivos trazados por una entidad, especialmente educativa.

La gestión es parte indispensable de un administrador educativo, especialmente cuando llega a una escuela en condiciones precarias, el Maestro Afre inicia concretizar a sus archivos administrativos, en donde registra cada acción en favor del centro educativo, al buscar fondos para comprar un sello exclusivo de la dirección; el cual serviría para más propiciar diferentes servicios educativos para los integrantes de la comunidad educativa de la instancia escolar; en donde se instituye varios caminos que forjan apoyo para los niños y niñas de la entidad en mención.

La comunicación con los padres de familia permite la organización de diferentes actividades, gestiones, afrontar retos y desafíos que en un centro educativo vive a diario; el permitirá consolidar la unidad de los integrantes de la comunidad escolar, especialmente en tiempos adversos como el conflicto armado interno del país, ejercer participación ciudadana en diferentes eventos de sonde viven y establecer ideales en base a las realidades de su modo de vida.

Para minimizar el flagelo social de pobreza será mediante la educación, porque permite fomentar en los usuarios ideas, herramientas de vida para propiciar cambios radicales principalmente en pensamientos neocoloni- 
zados; en donde narra la realidad de un contexto sumido en la pobreza extrema al ver llegar a los niños descalzos, botas de hule, zapatos de plásticos a consecuencia de los años de guerra interna, en donde el municipio de Nebaj fue un foco de muertes extrajudiciales en denota la realidad socioeconómica al ver a los niños llegar con ropa rota, sin suéteres en un lugar de extremo frío, por lo que el director y los docentes de la escuela iniciaron a enseñar a los niños a remendar su vestuario, ya que a la población le interesaba vivir en paz.

Como administrador educativo, la visión es fundamental en equipar de personal docente de forma permanente porque al momento de no tener a los docentes de cada grado o áreas de aprendizaje, retrocede al tener que esperar la reubicación de mentores provoca largos meses o días de espera; por lo que el Maestro Afre, gestiona plazas 011 en 1998, para consolidar un equipo de trabajo completo y tener una organización escolar de forma anticipada para no desamparar a los niños y niñas de la escuela.

El buscar recursos materiales especialmente didácticos es muy elemental para desarrollar el proceso de enseñanza-aprendizaje; principalmente al abordar diferentes temáticas, sin dejar a un lado la interacción de los estudiantes con los docentes, ya que mediante el tacto los niños y niñas fomentan un aprendizaje significativo. En donde la educación estética forma parte integral, un elemento muy necesario en cada área de aprendizaje de cualquier nivel educativo, a fin de consolidar el perfil de egreso de los usuarios de cualquier centro escolar.

Durante los años de conflicto armado interno, al ser protagonistas de un hecho histórico que afectaría de forma integral la estabilidad de las mayorías poblacionales del país guatemalteco, varías personas hasta la fecha sufren de recuerdos psicológicos de un evento aterrador; en donde la educación no fue prioritaria en muchas familias, si era necesario migrar al área urbana o movilizarse a otros lugares, incluso a otros países con resguardar la seguridad de los seres queridos era lo importante. Por lo que el Maestro Afre inicia procesos de apoyo psicológico en su ámbito de trabajo porque se coordinó trabajos con UNICEF y ASAUNIXIL, con un programa de salud mental quien lo dirigía el psicólogo Danilo Argueta, con un carisma y calidad de ser humano del profesional permitió la aceptación del magisterio nebajense, al propiciar ayuda a la población educativa en mención. Gestionar en diferentes entidades para dar alimentación a los niños de la escuela, al evidenciar de manera latente los niveles de desnutrición, mediante la refacción escolar generó un acto de humanidad a favor de los pequeños con sonrisas al minimizar de manera esencial el flagelo social de pobreza, secuelas especificas del contexto histórico de la nación; en donde la educación o los líderes con proyección social, contribuyen a propicia acciones más allá de una obligación de un servidor público. Gracias al apoyo de instituciones adicionales al Ministerio de Educación como el Comité Reconstrucción Nacional y Fundación contra el hambre se adquiere insumos necesarios para proporcionarles a los niños y niñas un vaso de atol en los días lectivos de clases.

Fomentar proyectos escolares permite forjar valores en los niños y niñas, por lo cual el Maestro Afre inicia a practicar diversos proyectos dentro de la escuela como coordinar actividades de jardinización del establecimiento educativo con el apoyo de don Jacinto como parte del personal operativo del centro escolar, docentes y la comunidad educativa, no escatimaron esfuerzos para dejar el establecimiento con vida a la infraestructura física de la entidad escolar permitió mediante la casita de madera de pajaritos, 
la disposición de realizar de forma incondicional un proyecto de esta naturaleza. El cuidar del corderito Panchito fue un proyecto que le agrado a los niños y niñas, ya que se turnaban para cuidarlo, lo que propició actitudes de responsabilidad, organización y valores humanos dentro de actividades extraescolares exitosas para consolidar el perfil de egreso de los estudiantes.

Como parte del magisterio nacional las actividades son elementales para la convivencia de mentores, como antesala de la celebración del día del maestro siempre se selecciona a directivos para distintas actividades académicas, culturales, deportivas y sociales que permiten salir de la monotonía cotidiana, el Maestro Afre fue elegido presidente de los juegos escolares en el año 2005 con diversas luchas se hizo realidad el mismo. Hay que indicar que en ese momento surgió otro movimiento magisterial en donde varias escuelas se retiraron para ser parte de las huelgas; en donde se fraccionó el magisterio de Nebaj por ideales muy de interés de los profesionales, situación que tambaleó la organización del evento, pero con el liderazgo, se logró sacar avante la actividad con apoyo de la municipalidad, el cual fue exitoso interactuar con el magisterio de Cotzal.

Educación inclusiva ya se en practicaba en la escuela, por ello se gestiona una silla de ruedas para el estudiante Roberto; porque tenía problemas para caminar y la importancia de apoyar a estudiantes con capacidades diferentes es un factor muy poco importante para el Ministerio de Educación, cuando en las políticas educativa se da publicidad de la misma, la realidad es otra; en primera instancia hay necesidad de incorporar herramientas para la atención de diversos estudiantes porque tiene ese derecho a estudiar y superarse. Con la iniciativa del Maestro Afre director del establecimiento educativo buscó medios para adquirir una silla de ruedas al estudiante, conjuntamente con una beca del estado que la cartera educativa proporciona en educación especial, por situaciones muy personales del estudiante no culmino sus estudios, pero es emprendedor $y$ lucha continuamente para vivir.

Forjar la educación tecnológica en la escuela de Xolacul, no fue tarea fácil porque en el gobierno de Oscar Berge vivió el magisterio una pugna muy directa con la Ministra María del Carmen Aceña, en donde impulsó las famosas escuelas demostrativas del futuro, con poca aceptación de varias escuelas del magisterio nacional, ya que las políticas del $\mathrm{Mi}$ nisterio de Educación se enfocaban en tener leyes macro no aceptadas por el gremio magisterial, al no tener claridad de las política del gobierno en mención, hizo que directores gestionaran un proyecto prometedor. En este caso el Maestro Afre no quedo atrás, la escuela donde dirigía fue seleccionada en el proyecto novedoso, con responsabilidades de la comunidad educativa en mención.

La gestión de internet para el aula demostrativa del futuro y desarrollar las famosas tics, se promovió con los padres de familia la autogestión permite la cuota mensual de un recurso indispensable para la docencia y los estudiantes, el cual permite a los usuarios tener un servicio no solo en la educación tecnológica, sino proveerle a los docentes insumos electrónicos para interactuar con un presente venidero de la sociedad actual y del conocimiento. Al final hay recompensa al realizar labores en bienestar del bien común, por lo que el director Afre fue seleccionado para viajar a Corea del Sur de parte del Ministerio de Educación, que permitió compartir con otros docentes del país e interactuar; en donde observó la educación de otra cultura, especialmente en la formación tecnológica del sistema educativo de dicho país asiático. 
Sumar esfuerzos para forjar extensiones universitarias de la Facultad de Humanidades como parte de la extensión, ya que en los años noventa solo en Santa Cruz del Quiché se tenía con el servicio de educación superior estatal, pero al iniciativa del Maestro Afre era descentralizar los servicios educativos para el municipio de Nebaj por lo que hizo los necesario para abrir una sección para el pueblo ixil; de igual forma fue participe la extensión del Centro Universitario de Occidente de la Universidad de San Carlos de Guatemala para preparar profesionales de la educación media del municipio; por la creación y funcionamiento del Centro Universitario de Quiché en el año 2010 el primer director el Lic. Marco Vinicio Marroquín Rosas tuvieron acercamientos para concretizar la primera cohorte de Licenciatura en Pedagogía y Técnico en Administración Educativa con Orientación en Medio Ambiente.

El Centro Universitario de Quiché vive una serie de problemas entre el año 2012, pero al llegar el Lic. Marco Vinicio de la Rosa Motepeque se establece una sección municipal para servir a la población nebajense, en donde él Maestro Afre es coordinador de la carrera hasta la fecha; por lo que no se cansa de abrir brechas a favor de una sociedad con un entorno histórico marcado por flagelos sociales, que actualmente se tratan de minimizar mediante el proceso educativo.

En la cuarta parte del libro el Maestro C. Fernando Afre Arévalo comparte las cartas de su renuncia para jubilarse ante el Ministerio de Educación, en donde expresa al ente del sistema educativo a mejorar muchos procesos en pro de la población guatemalteca, con el fin de brindar un servicio efectivo a los mismos; principalmente cuando en el gobierno de Berge suprimió plazas de supervisores educativos al reubicar docentes en el puesto, baja la calidad administrativa por la poca experiencia en el cargo; en donde deja un reflexión para realizar acciones educativas en favor del bien común.

La carta que dirige al director departamental de educación de Quiché hace una reflexión para garantizar la contratación del personal 021 de docentes, ya que la estabilidad laboral de los mismos no tenía garantía, especialmente con los pagos atrasados y sobre la exigencia no había pertinencia en la exigencia de los maestros de contrato, especialmente con el trato inferior que se les da, cuando todo mentor educativo posee las mismas funciones, atribuciones y responsabilidades como servidores públicos para ejercer la tarea docentes en las distintas comunidades del departamento de Quiché y por lo tanto la fragmentación del gremio magisterial no viene al caso.

\section{Juicio valorativo y crítico}

Este libro tiene un objetivo principal hacer una reflexión de la tarea docente con vocación, es una novela real que viven las comunidades educativas del sector público principalmente en el área rural donde las condiciones precarias repercuten en la organización escolar. El liderazgo del director es parte elemental de un centro escolar para realizar diferentes gestiones a favor de la comunidad educativa, la visión e integración de los partícipes del hecho educativo garantiza los cambios significativos ante cualquier escenario histórico que viva un contexto.

Cada acción educativa que el autor presenta en su obra concretiza actitudes muy funcionales desde la dirección de un centro educativo, tarea docente, apoyo a la comunidad y sociedad, al permitir incursionar el verdadero desarrollo social de un contexto social diverso como la nación guatemalteca. Al practicar una actitud propositiva germinará significativamente cambios de fondo a un 
que a pasos lentos. Esta herramienta educativa es un referente del como ejercer la labor educativa en cualquier nivel o sector del sistema de educativo nacional desde el nivel educativo preprimario, primario, medio e incluso a nivel superior; porque los procesos se asemejan en función de brindar un servicio a sus usuarios, a fin de entregar a la sociedad seres humanos que contribuyan a solucionar problemáticas de su entorno social.

Interesante conocer la disposición de un administrador educativo de carrera no se resistió al cambio, con el hecho de fomentar y gestionar un aula tecnología para en centro educativo responsable, dio una antesala a lo que venía en el 2020; al practicar una docencia y administración educativa diferente a la cotidianeidad, derivada por la pandemia COVID-19, que vino a revolucionar cada contexto de Guatemala, especialmente en comunidades rurales del país, en donde su realidad es diferentes a centro educativos privados . El tener una antesala de enfoque pedagógicos de educación a distancia, virtual y online, permite a los actores de la comunidad educativa a continuar con sus labores sin escatimar esfuerzos al momento de tener obstáculos en un proceso educativo diferente; especialmente cuando los entornos sociales son diferentes en cada región del país.

El tener políticas propias de la institución pertinentes a los que el Ministerio de Educación impulsa, permite a todo establecimiento educativo tener un proyecto educativo institucional, congruente a las demandas y necesidades de un contexto multiétnico, multilingüe y pluricultural, en función de establecer una docencia integral, sin omitir los cambios significativos de la sociedad del conocimiento e incorporarlos a la vida de los ciudadanos, sin olvidar la raíces ancestrales de cada lugar de la bella nación guatemalteca.
El compartir experiencias reales de la administración educativa en el presente escrito, proporciona un manual de vida para el quehacer educativo diario desde cualquier esfera del conocimiento y ámbito de que hacer educativo, ante cualquier época historia, escenario social; el cual permite la autoformación de los involucrados del proceso de formación. Por lo tanto, todo servidor público se debe a la población y por ende debe responder de manera significativa al tener a seres humanos valiosos desde su cosmovisión, axiología, riqueza natural y cultural de un municipio con reservas naturales interesantes, pero con la necesidad de favorecer a su población mediante la educación; principalmente en escenario caótico para la sociedad actual en donde fomentar una educación virtual se dificulta en el sistema educativo nacional, especialmente del sector público del país.

Por lo cual ante una nueva forma de lidiar procesos educativos en las repercusiones del COVID-19, mediante la lectura de este libro permitirá desarticular de la mente de los lectores una forma hegemónica de ver la educación como un factor bancario o monotonía laboral sin un recurso humano favorable a su entorno social, especialmente ante acontecimientos emergentes del mundo de hoy.

\section{CONCLUSIÓN}

Para ejercer la labor educativa los más importante es la vocación y disposición de servir a la población sin estereotipo alguno, más aún ante la situación educativa actual del país; que tiene dificultades para atender a la niñez principalmente del sector público al bridar el servicio educativo en tiempos de pandemia. Por lo cual el presente libro proporciona herramientas puntuales para afrontar los desafíos de los responsables de dirigir el hecho educativo en base a las polí- 
ticas educativas del país, demandas contextuales y emergentes que permitan formar a seres humanos proactivos, propositivos, productivos en pro del bienestar común, para impulsar la ciencia y tecnología en cualquier ambiente escolar.

\section{REFERENCIAS}

Afre.C.F (2016). Memoria de acción educativa. Santo Tomás Chiché, Quiché. ARTYK.

Constitución Política de la República de Guatemala (1985 con reformas de 1993). Congreso de la República.

Declaración Universal de los Derechos Humanos,10 de diciembre de 1948.

\section{SOBRE AUTORA}

\section{MSc. Viviana Reynoso Tavico}

Es Licenciada en Pedagogía y Administración Educativa, FAHUSAC-Quiché, Maestría en Docencia Universitaria, CUNOC-USAC, Doctoranda en Investigación en Educación del CUNOC-USAC; Profesor Interino de FID-Primaria Bilingüe Intercultural sección Santa Cruz del Quiché, Coordinadora de la Unidad de Investigación del Centro Universitario de Quiché, CUSACQ

\author{
Conflicto de intereses \\ Declara no tener ningún conflicto de intereses. \\ Declaración de consentimiento informado \\ El estudio se realizó, respetando el Código de ética y buenas prácticas editoriales de publicación. \\ Derechos de uso \\ Copyright $\odot 2021$ por Viviana Reynoso Tavico \\ Este texto está protegido por una licencia Creative Commons 4.0. Internacional
}

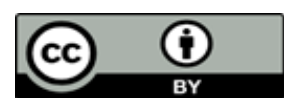

Usted es libre para compartir, copiar y redistribuir el material en cualquier medio o formato y adaptar el documento, remezclar, transformar y crear a partir del material para cualquier propósito, incluso comercialmente, siempre que cumpla la condición de atribución: usted debe reconocer el crédito de una obra de manera adecuada, proporcionar un enlace a la licencia, e indicar si se han realizado cambios. Puede hacerlo en cualquier forma razonable, pero no de forma tal que sugiera que tiene el apoyo del licenciante o lo recibe por el uso que hace. 